\title{
Calculating the Fractal Dimension of the Material Fracture Surface Based on the Triangular Prism Surface Area Method
}

\author{
Yu Gong, Chengbao Wu, Jian Wang, Lei Kong \\ School of Aircraft Maintenance and Engineering, Guangzhou Civil Aviation College, Guangzhou \\ 510430, China,
}

Keywords: Triangular prism surface area method; fractal; fractal dimension

\begin{abstract}
Fractal dimension is one of the parameters for characterizing the fracture surface of materials quantitatively, and is also the basis for analyzing the fracture mechanism of materials, so it is very important in mechanic of materials. In order to improve the efficiency of calculation on fractal dimension of fracture surface of materials by triangular prism surface area method (TPSAM), a program for TPSAM based on Matlab 7.1 was compiled and verified by using of two different samples. The results indicated that the program was easy to be read and operate, and the calculated fractal dimension of the samples was in a range from 2 to 3. Therefore, the program was accurate and could be used widely.
\end{abstract}

\section{Introduction}

The mechanical properties of material are determined by its internal micro structure and experimental conditions, and the micro structure and the experimental conditions also affect the morphology of fracture surface of material. The researchers could study the relationship between the micro structure and the mechanical properties of the material by analyzing the characteristic of the fracture surface of the material. Therefore, how to characterize the morphology of the fracture surface of the material correctly and effectively was taken attention widely for a long time, and the researchers put forward many characteristic parameters. The parameters could be divided into three types. The first type is the qualitative characterization by the terms of the smoothness, flatness, coarseness, etc., thereby the fracture mechanism of material could be described as the brittleness, toughness and the combination with brittleness and toughness. The second type is statistic parameters including two dimensional parameters based on surface profile curve and the statistics and the three dimensional parameters based on the statistics [1]. The third type includes the roughness index including the roughness indexes of the profile curve of fracture surface and the ones of the fracture surface [2]. The parameters mentioned above had shortcomings of themselves. For example, the quantitative parameters can not evaluate the objective characteristics of the morphology of the fracture surface of the material, the number of the traditional statistic parameters and the roughness indexes of the fracture surface is too large and they are unstable [3].

The fractal theory was founded at the end of the 70s and early 80s in last century by Mandelbrot. The theory can be used to observe the fine structure hidden in the confused phenomena by using the similarity principle. So it is a new method for people to comprehend the world from the locality to the global. In 1984, Mandelbrot proposed firstly that the morphological characteristic of the fracture surface of the metal material could be described by the fractal theory [4]. With more than 30 years' development, the fractal theory had been used to characterize the engineering surface, interface, and the the morphological characteristic of the fracture surface of the different material quantitatively [5 11], and at the same time, many methods used to measure the fractal dimension were proposed, which can be divided into two classes. The first one is indirect measurement method including the Secondary Electron Beam Scanning Method(SEBSM), the Vertical Section Method (VSM), and Silt Island Method (SIM), the second one is direct measurement method that includes the Pixel Covering Method (PCM) [12-13], The Projective Covering Method (PCM) [14] and Triangular Prism Surface Area Method (TPSAM) [15] etc..Among them, the TPSAM is earliest method and is widely used based on the grey image. Many researchers wrote the calculating procedures for the method to improve the calculating efficiency based on the C procedure language [16-17]. But, the 
source codes of the $\mathrm{C}$ procedure language is too long, always more than 300 procedure statements, and its operation efficiency is relative low [15].in this paper, the authors wrote the calculating procedures based on the Matlab 7.1 for shortening the procedure statements and improving the operation efficiency.

\section{The basic principle of TPSAM}

The measuring principle was show in Figure 1(a) and Figure 1(b). The sign A and B in Fig. 1 represents the true fracture surface and projective network covering the true facture surface respectively.

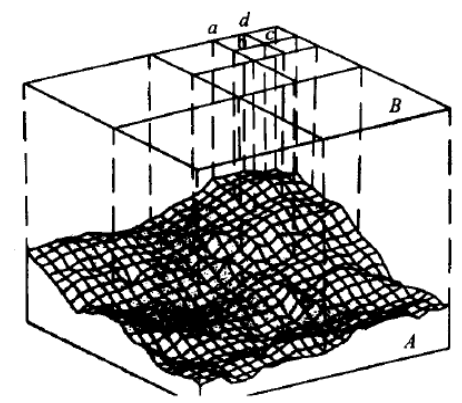

(a)

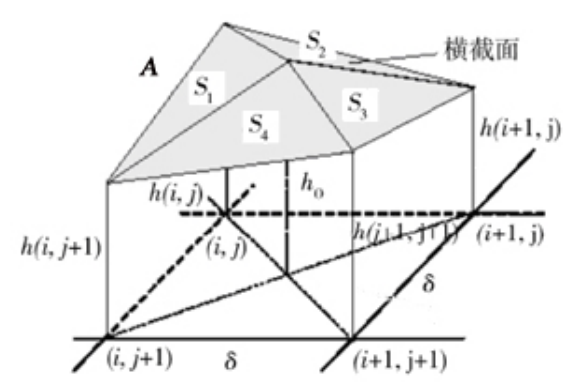

(b)

Fig.1 the TPSAM: (a) The projective network, (b) the projective grid

If one chooses the grid abcd ( $a, b, c, d$ point is the vertex of the grid respectively) with size $\delta^{*} \delta$ to cover the facture surface at $k$ time, and $h(i, j+1), h(i+1, j+1), h(i+1, j)$ and $h(i, j)$ are the height of the points a, b, c and d, respectively (as the Fig.1b shows). Four triangles S1, S2, S3, S4 are used to instead of the true coarse morphology of abcd. Then one can calculate the area Sij of the coarse surface enclosed by the projective grid of the abcd. If the number of the projective grids is $N(\delta)$ and to calculate the surface area of the zone $\delta * \delta$, one gets the total surface area[15] :

$$
S(\delta)=\sum_{i, j=1}^{N(\delta)} S_{i, j}
$$

Then, the fractal dimension of the fracture surface can be calculated by the following equation [15]:

$$
S(\delta) \propto \delta^{2-D s}\left(2 \leq D_{s} \leq 3\right)
$$

If taking a bi-logarithmic system for the Eq.2, fitting the data linearly, and letbe the slipe of the linear $\alpha$, one can get:

$$
D_{s}=2-\alpha
$$

\section{The calculation method based on Matlab 7.1}

\subsection{The theoretic fundaments of calculation}

In gray scale image, each pixel stands for a sampling color, and the image normally displays from the darkest black to the most the brightest white. In the corresponding matrix, a number from 0 to 255 is used to represents a pixel, 0 represents the color of black, and the 255 white. The Fig. 2 shows the one portion of the gray scale image, and corresponding matrix is shown in the Fig.3. 


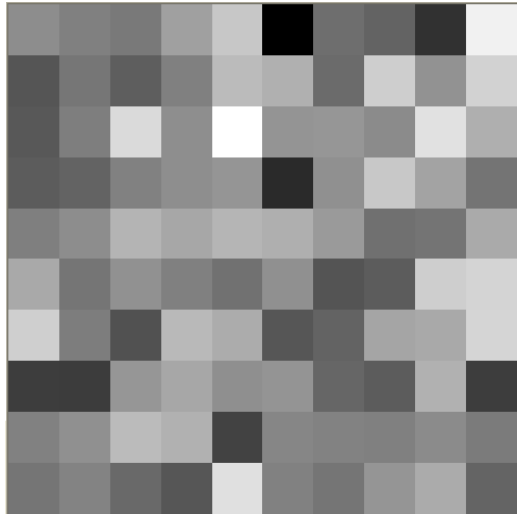

Fig .2 gray scale image

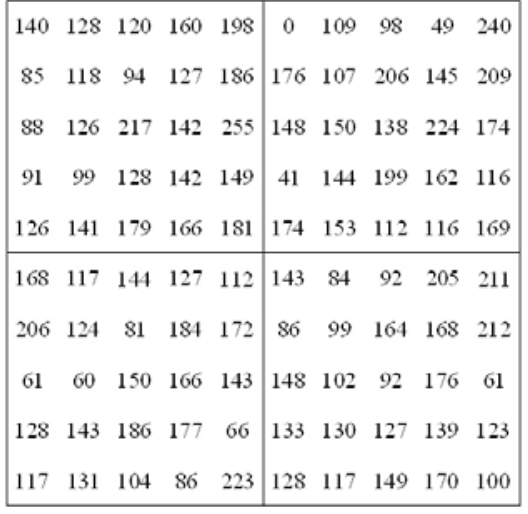

Fig.3 matrix of the gray-scale image

When the TPSM is used to measure the fractal dimension of the gray scale image, the size of the projective grid (as in Fig. 1(a) shows) can be instead of by the number of pixels. For example, the pixel matrix $5 * 5$ represents a projective grid; to change the size of the projective grid can be realized by changing the dimension of the pixel matrix. As calculating in the computer, one can extract the four vertexes of the each matrix $5 * 5$ firstly, and then gets the surface area of each projective grid $\mathrm{N}(5)$ and the whole surface area $\mathrm{S}(5)$ of all of the matrixes $5 * 5$. If the dimension of the pixel matrix is 10 , the number of projective grid is $\mathrm{N}(10)$, and the whole surface area is $\mathrm{S}(10)$. If the dimension of the pixel matrix is $\delta$, the number of projective grid is $\mathrm{N}(\delta)$, the whole surface area is $\mathrm{S}(\delta)$.Lastly, one can get the fractal dimension according to Eq.2 and Eq.3.

\subsection{Calculation steps}

The block diagram for the calculation program for surface fractal dimension by TPSAM is shown in Fig.4.

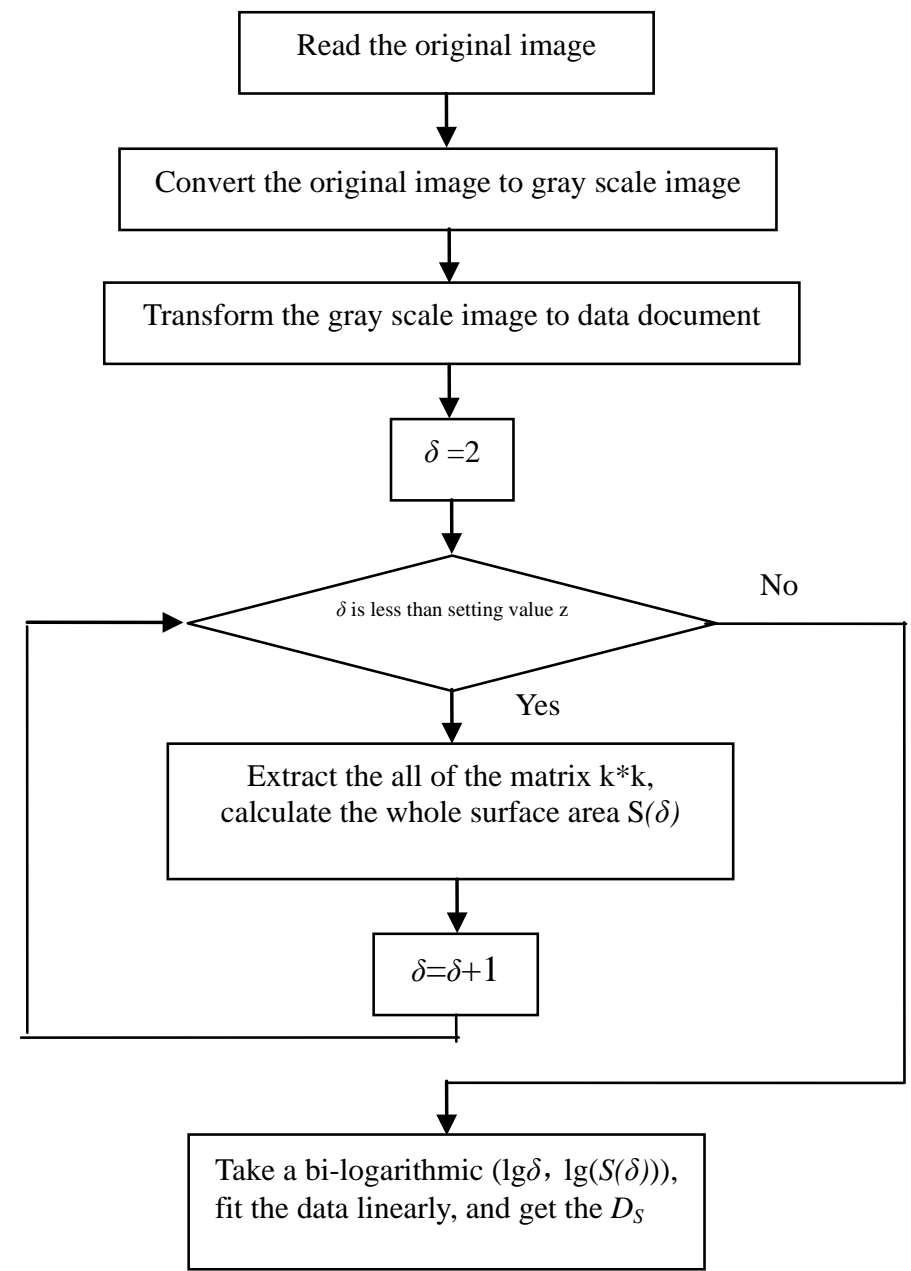

Fig.4 the calculation program for surface fractal dimension by TPSAM 
The main steps are following:

Step1: Input the image took by the Scanning Electron Microscope (SEM) into the computer.

Step2: Read the image by the software Image Pro-Plus (IPP).

Step3: Delete the irrelevant information in the image such as the label, the ruler, and the date, and so on.

Step4: Open the Matlab 7.1 and write the procedure according to the principle of the TPSAM.

Step5: Simplify the procedure and calculate the fractal dimension.

\section{Case study}

The procedure based on the Matlab 7.1 was used to calculate the fractal dimension of fracture surface of two different material samples (sample 1 and sample 2) in order to verify its accuracy. The SEM images of fracture surface of the different materials were shown in Fig.5 (a) and (b).

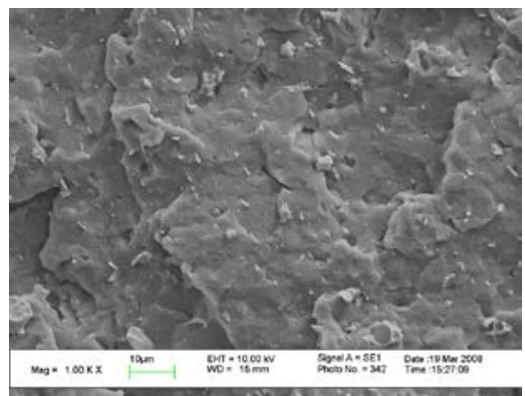

(a)

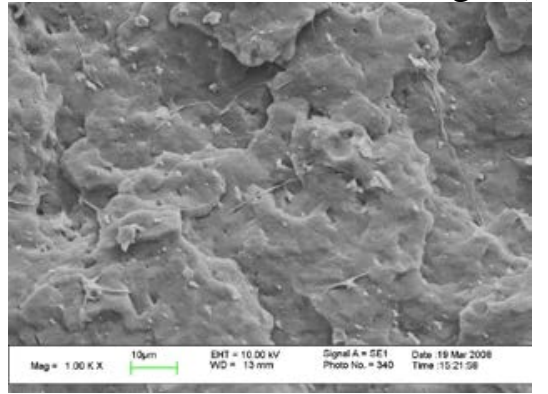

(b)

Fig.5 the SEM images of fracture surface samples 1 and 2: (a) sample 1, (b) sample 2

Early in 1978, Sayles believed that the surface morphology is a non-stationary random process. From Fig.5 (a) and (b), we could find that the morphology of the fracture surface of sample 1 and 2 is not flat. That is to say, there are so many ups and downs. So, the traditional statistic parameters could not characterize the randomness and the detail feathers. The parameter fractal dimension makes it possible that the fracture surface toughness can be characterized quantitatively, and the difference among the different fracture surface can be distinguished easily [5, 12-15]. So, the fracture surface fractal dimensions of sample 1 and 2 were calculated by using the calculation steps in Fig.4. When the setting value $\mathrm{Z}$ is 100 , the calculated curve of fractal dimension of fracture surface of sample1 and 2 were shown in Fig.6 (a) and (b):

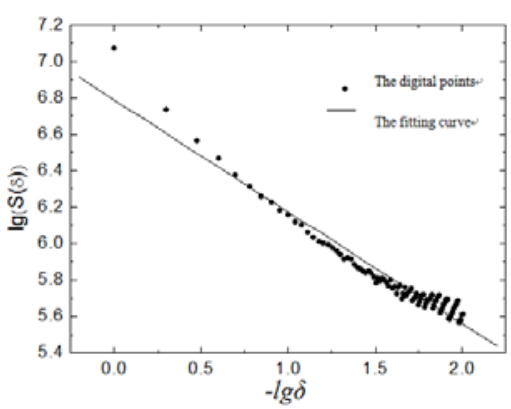

(a)

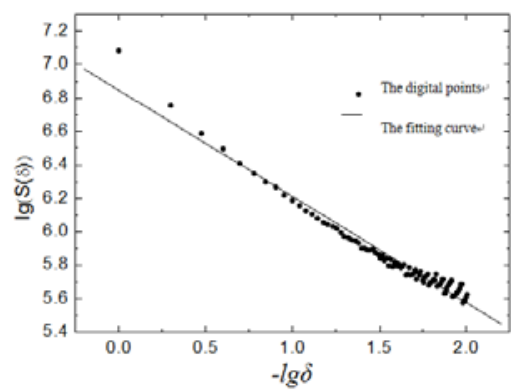

(b)

Fig.6 the fractal dimensions of samples 1 and 2 as $\mathrm{Z}$ is 100: (a) sample 1, (b) sample 2

It can be seen from Fig. 6 (a) and (b) that the digital points $(-\lg \delta, \lg S(\delta))$ are almost linear. In order to investigate the fractal characteristic of the fracture surface of the material at the different level, the paper considered the relationship between the fractal dimensions, correlation coefficients $\mathrm{R}$ of least squares linear regression analysis and the setting value Z, as Fig.7 (a) and (b). 


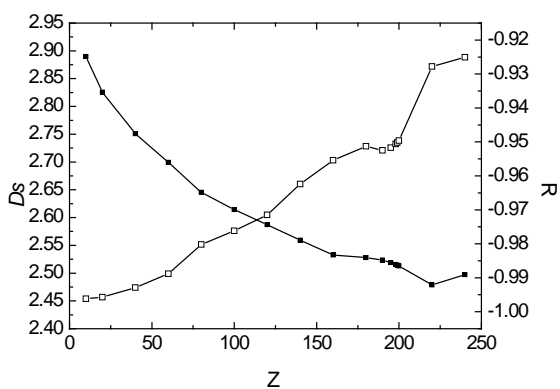

(a)

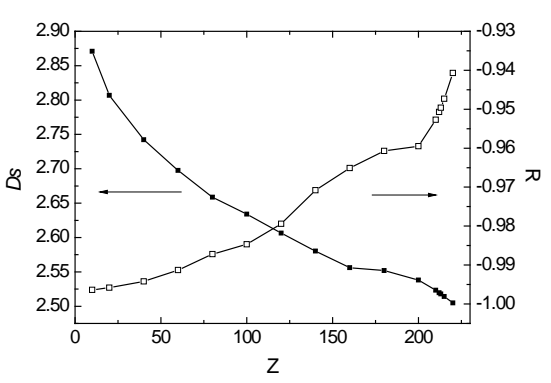

(b)

Fig.7 the fractal dimensions of samples1 and 2 at different $Z$ value: (a) sample 1, (b) sample 2

The Fig.7 (a) and (b) indicated that the calculated fractal dimension is in a range from 2 to 3.that is to say, the procedure wrote in this paper based on the Matlab 7.1 could calculate the fractal dimension accurately. From the Fig.7 (a) and (b), we could also know that the fractal dimension and the $\mathrm{R}$ decreased with the increasing of the setting value $\mathrm{Z}$, and as the $\mathrm{Z}$ is larger than certain number, the $\mathrm{R}$ is less than 0.95.this is reason that fractal characteristic of the fracture surface and engineering surface is limited, when calculating the fractal dimension, if the measuring size is less or larger than a certain value, the calculating results would deviate the true value [15-18].

\section{Conclustion}

The procedure was easy to be read and operate, and the calculated fractal dimension of the samples was in a range from 2 to 3. Therefore, the program was accurate and could be used widely.

\section{References}

[1] Thomas T.R., Characterization of surface roughness. Precision Engineering, 3(2), pp97-104, 1981.

[2] El-soudani S.M., Theoretical basis for the quantitative analysis of fracture surfaces. Metallography, 7, pp271-311, 1974.

[3] Liang J.Z., Wu C.B., A fractal model of tensile fracture surfaces for particulate-filled polymer composite. Journal of Applied Polymer Science, 109, pp3763-3767, 2008.

[4] Mandelbort B. B., Fractals: form, chance, and dimension. San Francisco: Freeman, 1977.

[5] Mandelbort B. B., The Fractal Geometry of Nature. San Francisco, Freeman, 1982.

[6] Mandelbrot B.B., Passoja D.E., Paulla A.J.. Fractal character of fracture surface of metals. Nature, 19, pp721-722, 1984.

[7] Bigerelle M. Iost A., A new method to calculate the fractal dimension of an interface application to a Monte Carlo diffusion process. Computional Material Science, 24, pp122-127, 2002.

[8] Antonio T., Conrad J.P., Multifractal geometry in stock market time series. Physica A, 322, pp629-649, 2003.

[9] Jia Xiao-qiang, Fang Xiang, Ning Qiang, Pan Jun, Xiao Jie-shan, New Grayscale Method of Calculating 3-dimensional Fractal of Rock Section. Blasting, 29(2), pp38-41, 2012,.

[10] Feng Jie, Tan Yun, Tao Ping, Fan Ying, Niu Wei, Chen Yongme, Effects of Inner Hydrogen on Fracture Fractal Dimension of Austenitic Stainless Steels Tensile Specimens. Materials Review, 28(2), pp118-122, 2014.

[11] Zheng Jin-ling, Hu Xiao-fang, Characterization on Mechanical Properties of Mortar by Fracture Fractal Dimension. Materials Review, 25(6), pp139-142, 2014.

[12] Tang Wei, Zhu Hua, Wang Yong, Fractal Behavior of Stress Corrosion Fracture. Journal of Iron and Steel Research, 19(8), pp56-58,2007.

[13] Zhang X. D., Meng Q. C., Multi-feature edge extraction with local fractal features. Conference on Convergent Technologies for Asia-Pacific Region, TENCON, pp247-251, 2003.

[14] Wang Jinan, Xie Heping, Tian Xiaoyan, etc, Direct Fractal Measurement of Fracture Surfaces. Journal of University of Science and Technology Beijing, 21(1), pp6-9, 1999.

[15] Clarke K. C., Computation of the fractal dimension of topographic surfaces using the triangular 
prism surface area method. Computers and Geosciences, 12(5), pp713-722, 1986.

[16] Sayles R. S., Thomas T. R., Surface topography as a non-stationary random process. Nature, 271, pp431-434, 1978.

[17] Stachowiak G. W., Podsiadlo P., Characterization and classification of wear particles and surfaces. Wear, 249, pp194-200, 2001.

[18] Wang Y. L., Du B.Y., Liu J., et al, Surface analysis of cryofixation-vacuum-freeze-dried polyaluminum chloride-humic acid (PACl-HA) flocs. Journal of Colloid and Interface Science, 316, pp 457-466, 2007. 